\title{
The psychophysics of absolute threshold and signal duration: A probabilistic approach
}

\author{
Ray Meddis ${ }^{\text {a) }}$ and Wendy Lecluyse \\ Department of Psychology, University of Essex, Wivenhoe Park, Colchester, Essex, CO4 3SQ, \\ United Kingdom
}

(Received 17 July 2010; revised 18 February 2011; accepted 25 February 2011)

\begin{abstract}
The absolute threshold for a tone depends on its duration; longer tones have lower thresholds. This effect has traditionally been explained in terms of "temporal integration" involving the summation of energy or perceptual information over time. An alternative probabilistic explanation of the process is formulated in terms of simple equations that predict not only the time/duration dependence but also the shape of the psychometric function at absolute threshold. It also predicts a tight relationship between these two functions. Measurements made using listeners with either normal or impaired hearing show that the probabilistic equations adequately fit observed threshold-duration functions and psychometric functions. The mathematical formulation implies that absolute threshold can be construed as a two-valued function: (a) gain and (b) sensory threshold, and both parameters can be estimated from threshold-duration data. Sensorineural hearing impairment is sometimes associated with a smaller threshold/duration effect and sometimes with steeper psychometric functions. The equations explain why these two effects are expected to be linked. The probabilistic approach has the potential to discriminate between hearing deficits involving gain reduction and those resulting from a raised sensory threshold. (C) 2011 Acoustical Society of America.
\end{abstract}

[DOI: $10.1121 / 1.3569712]$

PACS number(s): 43.66.Ba [CJP]

Pages: $3153-3165$

\section{INTRODUCTION}

The quietest sound that a person can reliably hear is often called his or her "absolute threshold." In fact, both words, "absolute" and "threshold," require qualification. The quantity is not absolute because it changes over time and depends upon the stimulus characteristics, particularly on the stimulus duration; longer sounds have lower thresholds. The term threshold is likewise misleading because it indicates a discrete dividing line between levels at which the sound can and cannot be heard. In reality, threshold normally describes the midpoint of a range of levels where the probability of detection varies between zero and one. The threshold for a tone is therefore both probabilistic and duration dependent. To add further complication, the nature of the dependencies is different across individuals and these differences are most evident in listeners with a hearing impairment. In this report, we explore the potential of a simple probabilistic explanation of these psychophysical effects to understand the phenomena.

The dependence of threshold on duration is traditionally explained in terms of an integrator that accumulates stimulus energy over time until some criterion value is reached. Shorter stimuli will therefore require more intense stimulation to reach the criterion. For example, Garner and Miller (1947) suggested that the detection system operates as a perfect energy integrator; when the integrated energy reaches a certain level, the stimulus becomes audible. Plomp and Bouman (1959) have characterized this relationship as $I=I_{\infty} /(I-\exp (-d / \tau))$, where $I$ is the intensity at threshold

\footnotetext{
a) Author to whom correspondence should be addressed. Electronic mail: rmeddis@essex.ac.uk
}

for a particular tone, $I_{\infty}$ is the intensity at threshold for a very long tone, $d$ is the duration of the tone, and $\tau$ is the time constant of integration. Essentially, this suggests that the detection system is like a low-pass electrical filter that sums energy using a "leaky integrator." When the contents of the integrator reach a certain value, detection occurs.

The first parameter, $I_{\infty}$, implies a minimum level of sensitivity which cannot be improved by increasing the duration of the stimulus. Licklider (1951) characterized this as the "diverted input hypothesis," and Green et al. (1957) described the parameter saying that "a certain portion of the stimulus intensity is not an effective stimulus for the ear." This is an interesting concept that appears to introduce a threshold inside an explanation of threshold. To avoid confusion, we shall use the term "sensory threshold" to refer to this minimum stimulus level while the term threshold will refer generally to the observed stimulus level required to guarantee a certain probability of detection.

The second parameter, the time constant of integration $\tau$, has been the subject of many quantitative investigations but has proved to be difficult to pin down. A systematic analysis of 50 human and animal studies by Gerken et al. (1990) found that estimates of $\tau$ varied considerably between 46 and $588 \mathrm{~ms}$ depending on tone frequency, stimulus type (e.g., tones and noise), and any hearing impairment.

Green et al. (1957) described their own data using a "power law" which identifies the threshold as $I=C d^{-m}$, where $I$ is the intensity at threshold measured relative to the sensory threshold and $d$ is duration as before, while $m$ and $C$ are free parameters determined from the data. The power law is defended on the grounds that it is a good fit to the data in the sense that threshold expressed in decibels is a straight- 
line function of time when duration is expressed on a logarithmic scale. However, Green et al. acknowledged as a matter of regret that the function was a good fit only if the exponent, $m$, was allowed to vary with duration. They concluded that "a rational theory is badly needed to explain why these or similar relationships exist."

The probabilistic nature of responding near threshold is most commonly explained with reference to a hypothetical internal source of unceasing Gaussian noise that masks the internal representation of low-level tones. This noise adds variability to detection decisions near threshold and justifies the practice of using signal detection theory (Green et al., 1957) to account for threshold data. Signal detection theory claims that the hearer specifies a best criterion level, $C$, of the internal activity and uses it to distinguish between "signal present" and "signal absent." The criterion, $C$, is another interpretation of the "sensory threshold" $\left(I_{\infty}\right)$ described above. Signal detection theory was originally developed to provide a basis for identifying signals that occurred against a background of physical noise. It is extended by Green et al. to include signals presented in quiet by assuming an internal (possibly physiological) noise. Altogether, this is a tripartite theory consisting of (1) continuously present internal noise, (2) an integrator, and (3) a decision procedure. It constitutes, for many, the canonical theory of absolute threshold.

The most successful model of this type (Viemeister and Wakefield, 1991) incorporates all of these features but decomposes the decision process into a series of successive short "looks" where each look generates an estimate of the likelihood that a tone is present. Successive estimates are aggregated across the duration of the stimulus to form the basis of a final decision. This accumulation of estimates is a different kind of integrator based on the accumulation of information but an integrator nonetheless. The "multiple looks" approach has the advantage of being able to function across stimulus presentations or across a range of sources of information; for example, across frequency channels (Hoglund and Feth, 2009). With "intelligent looking," this approach can also be made to ignore irrelevant information such as interspersed masking noises and silences.

From many points of view, these theories give a useful account of the nature of absolute threshold but there are doubts. First among these is the question of the physical nature of the integrator. Also, if there is a physical leaky integrator, why is it so difficult to identify its time constant of integration? If the power function is a good fit, why does the exponent need to change with signal duration? A different question concerns the nature of the unceasing internal noise that restricts our ability to detect low-intensity sounds. Its nature is rarely explicitly discussed beyond informal speculation that this is the amplitude of the noise associated with auditory nerve (AN) spontaneous activity.

Integrator theories have in common the idea that a detection event derives from a summary of the recent sensory history of the system, i.e., a memory. There is, however, an alternative type of theory based on probability accumulation over time that requires no memory. Probability theories have been considered and rejected from time to time by auditory theorists (e.g., Garner and Miller, 1947, and Zwislocki, 1960).
However, Watson (1979), a visual scientist, proposed a simple probabilistic account of the threshold/duration relationship that might prove more acceptable and his formulation will be used as the basis of the work to be reported below. He proposed that a quantal signal detection event could occur at any point in time during the stimulus and the probability of at least one detection event could be found by summing instantaneous probabilities across time using the general formula, $P=1$ $-\Pi\left(1-p_{t}\right)$, where $P$ is the probability of detecting the stimulus as a result of at least one detection event occurring during the stimulus and $l-p_{t}$ is the probability of not detecting the stimulus at time $t$. The symbol $\Pi$ represents the AND function in probability so that $\Pi\left(l-p_{t}\right)$ represents the probability of not detecting the stimulus at any time during the stimulus. His approach has some useful features. For example, no integrator or memory is required; the system simply waits for an event that might or might not occur at any time. Also, the probability of detection will be greater for longer stimuli.

The lack of a physical integrator is an attractive feature when efforts are made to identify the physiological substrate responsible for these processes. For example, the dependence of threshold on duration can be observed at a very low level in the auditory nervous system. Clock et al. (1998) have demonstrated the dependence of threshold on duration at the level of the AN. At an earlier date, similar observations were made in single-units in the cochlear nucleus (Clock et al., 1993). At the level of the AN or the cochlear nucleus, there is no physiological process capable of acting as a temporal integrator with an appropriately long time constant of integration summarized by Gerken et al. (1990). Heil and Neubauer (2003) identified this as a central paradox in our conceptualization of sensory threshold. However, they and Krishna (2002) both showed that a solution might be found (at least at the level of the auditory periphery) in terms of probabilities and they used AN first-spike latencies to illustrate the point.

Heil and Neubauer (2001) showed that AN first-spike latencies could be predicted by the integral of the stimulus pressure envelope prior to the first driven spike following stimulus onset. Thus, the system was behaving "as if" it were integrating pressure even though no physical integrator could be identified. When the level of the stimulating tone is low, the probability of a driven spike will also be low and the delay to the first spike will be greater. If the stimulus is too short, it may come to an end before a spike has occurred. They argue that the likelihood of observing a driven spike before the end of the signal can be equalized across stimuli with different levels only by adjusting the duration of the stimulus. Conversely, if the durations of the stimuli are different, the likelihood of obtaining a driven spike can be equalized only by changing the level. In other words, a threshold/duration (T/D) function is purely a consequence of delays to the first driven spike in AN fibers.

In their view, the parallels between these delays and psychophysical T/D functions were strong enough to justify an explanation of the psychophysical T/D effect in terms of first-spike latencies. They point to two parallels. The first is simply the demonstration that apparent temporal integration is taking place at the lowest level of the auditory system and the obvious inference that it must be involved in stimulus 
detection. The second parallel consists of redefining threshold as the pressure integral of the stimulus at the point when the first spike occurs and showing that this increases as a power function of signal duration in a manner remarkably similar to the T/D effect observed in psychophysical measurements. Their subsequent mathematical analysis of the first-spike latency data demonstrated that latencies could be understood in terms of low-probability events at the inner hair cell (IHC)/AN synapse (Neubauer and Heil, 2008).

At this point, we need to make a distinction between (a) their specific suggestion that the T/D function can be explained in terms of first-spike latency and (b) their general suggestion that probabilistic processes at work in the nervous system could form the basis of a (memory-less) explanation of thresholds. For example, they make the specific suggestion that the slope, $m$, of the power function relating duration and the pressure integral at threshold must be close to 0.667 and link this to the number of $\mathrm{Ca} 2+$-binding steps to the ca2+-sensor mediating release (Heil and Neubauer, 2003). Both general and specific hypotheses could be true but we restrict ourselves below to the more general account and formalize a purely psychophysical approach based on a stream of probabilistic events without reference to the underlying physiology. This will allow us to move forward with a psychophysical account while leaving some unresolved physiological problems for separate study.

One of these problems concerns the question of how the nervous system separates driven AN spikes from the continuous stream of spontaneous activity. Neubauer and Heil (2008) are aware of the problem and agree that this might be resolved later in the system using coincidence detection mechanisms such as those used in a computer modeling study (Meddis, 2006). If this point is conceded, it must also be accepted that the mathematics of coincidence detection might take precedence over the mathematics of first-spike latency. Another problem concerns their use of probabilistic explanations to describe their physiological data but use of the traditional power function to describe their psychophysical results. They justify the latter practice purely in terms of how well the function fits the data. This is the same justification used by Green et al. (1957) and leaves unanswered their request for a more rational basis. Below, we will suggest that the power function can be replaced by a different function with a clearer rationale.

Neubauer and Heil (2004) also considered threshold changes in hearing impairment. Garner and Miller (1947) had previously sought to explain threshold partly in terms of a sensory threshold specified in terms of a minimum signal intensity, $I_{\infty}$. Neubauer and Heil proposed a similar idea but expressed in terms of a sound pressure level (SPL), $P_{\text {ineff }}$, below which acoustic signals are ineffective. In their view, hearing impairment could involve either a reduction in the system gain or an increase in the sensory threshold or changes to both. They analysed and modeled T/D functions following acoustic trauma using data supplied by Solecki and Gerken (1990) and found changes in both. Below, we will continue to make their basic distinction between gain and sensory threshold, although our measure of sensory threshold will be different.
The issue is of practical importance because the measurement of absolute thresholds is central in the diagnosis of hearing loss and the prescription of prostheses. High thresholds are a symptom of pathology but we are often unable to say what that pathology is; the same high threshold could imply different underlying causes. A possible basis for differentiation lies in "brief-tone audiometry" where multiple threshold measurements are made at different tone durations (Elliott, 1963; Sanders and Honig, 1967; Richards and Dunn, 1974; Wright, 1978).

Individuals with normal hearing typically show a substantial reduction in thresholds for longer tones compared to short tones (e.g., Hughes, 1946; Plomp and Bouman, 1959; Sheeley and Bilger, 1964; Olsen and Carhart, 1966; Hall and Fernandes, 1983; Florentine et al., 1988). For example, the threshold for a $10-\mathrm{ms}$ tone may be $10 \mathrm{~dB}$ higher than for a 250-ms tone. In the case of hearing loss, thresholds will be raised for tones of all durations but the rise in threshold for longer tones is greater than for short tones (Wright, 1968, Watson and Gengel, 1969; Gengel and Watson, 1971; Hall and Fernandes, 1983; Florentine et al., 1988; Gerken et al., 1990). Gerken et al. (1990) found estimated T/D slopes to be as low as $3 \mathrm{~dB} /$ decade for some hearing-impaired listeners but also found some slopes as high as $10 \mathrm{~dB} /$ decade.

Attempts to use brief-tone audiometry to distinguish between different types of hearing impairment have a long history but the procedure has never become a standard clinical practice. For example, reduced T/D effects were found specifically in listeners with presbycusis, noise-induced hearing loss, cochlear hearing loss, and Meniere's disease, while listeners with a conductive hearing loss or an eighth nerve lesion appear to yield normal threshold-duration effects (Harris et al., 1958; Pedersen and Elberling, 1973; Young and Kanofsky, 1973; Olsen et al., 1974; Pedersen and Salomon, 1977; Chung and Smith, 1980; Chung, 1982). Unfortunately, the variability of the threshold/duration effect among both normal and impaired listeners and the overlap found between the two groups created considerable uncertainty concerning the clinical value of brief-tone audiometry. This may explain why it has not been adopted in clinical settings.

In what follows, the probability accumulation equation proposed by Watson (1979) will be used as the starting point to develop a small system of equations to represent a general formulation of a probability accumulation account of threshold. The final product however is different from Watson's approach but offers a number of advantages. First, it can be used very simply to generate a T/D function that fits the data at least as well as a simple power function but with the advantage of a more rational basis. Second, the same equation can be used to generate the psychometric function without reference to the unceasing noise required by signal detection theory. Third, this approach allows us to base our predictions on the simple principle of stochasticity without needing to commit to a particular physiological basis. A strong prediction from the model is that the psychometric function can be inferred directly from the T/D function and vice versa. This idea will be tested using measurements made using listeners with both $\mathrm{NH}$ and $\mathrm{IH}$. 
It will be argued that there is merit in routinely decomposing threshold measurements into two sub-components, "gain" and sensory threshold, particularly when assessing hearing impairment because the same threshold as measured by pure-tone audiometry may conceal differences along these two dimensions. Our approach will be neutral with respect to the underlying physiology but the discussion will consider similarities and differences with recent theorising in this area.

\section{STATISTICAL MODEL}

The hypothesis is based on the simple concept that acoustic stimulation gives rise to a single stream of stochastically distributed internal detection events and that the rate of occurrence of these events is proportional to the tone peak pressure. They are defined as "detection events" because the occurrence of a single event is enough to give rise unambiguously to detection.

At low signal levels, these events occur at a low rate and, given their stochastic nature, it is possible for a short tone to begin and end without any event occurring. If this happens, the presence of the tone will not be detected. However, a longer tone at the same level offers a more extended opportunity for at least one detection event to occur. If the levels of a long and a short tone are adjusted to equate the probability of at least one detection event occurring during the tone, the longer tone will necessarily be set at a lower level. In this way, lower thresholds for longer duration can be understood without reference to a temporal integrator. In the following equations, the detection events are equally likely to occur at any time during the stimulus. This is similar to the claim of Heil et al. (2008) that their model predicts that "the stimulus generates a constant probability of exceeding the AN fiber's detection threshold when the stimulus amplitude is stationary."

This probabilistic approach can be formalized simply. The main equation determining the rate of events, $r$, is $r=G P$, where $P$ is the peak pressure of a pure tone $(\mu \mathrm{Pa})$ and $G$ is a free parameter representing the gain of the system (events $/ \mathrm{s} / \mu \mathrm{Pa}$ ). Where stimuli are ramped, $P$ is defined as the mean of successive peak pressures, i.e., the mean of the envelope of the stimulus.

However, there is an internal absolute limit to the system's sensitivity. We incorporate this idea into the rate equation by adding a second parameter, $A$,

$$
\begin{aligned}
& r=G P-A \text { for } G P>A, \\
& r=0 \text { for } G P<=A,
\end{aligned}
$$

where $A$ is a rate of events below which the system does not ever respond and might be called the "discounted rate" in recognition of Licklider's (1951) diverted input hypothesis.

\section{A. Psychometric functions}

From basic probability theory, we can specify the probability, $\Psi$, that at least one detection event will occur between the beginning and the end of the tone

$$
\Psi=1-\exp (-d r)
$$

where $d$ is the duration of the tone (in seconds). This is a version of Watson's (1979) equation described in the Introduction. Substituting for $r$ [see Eq. (1)] gives

$$
\Psi=1-\exp (-d(G P-A))
$$

Equation (3) is the psychometric function, i.e., the probability that a tone will be detected as a function of tone level.

Figure 1 illustrates Eq. (3) using four different tone durations $(32,64,128$, and $256 \mathrm{~ms})$. Fixed values for parameters $G$ and $A(0.04$ and 2$)$ are chosen for the purpose of illustration to give thresholds in the region of $10 \mathrm{~dB}$ SPL. The functions are shown twice in Fig. 1. The left panel uses micropascals as the $x$-axis so that the relationship with Eq. (3) is transparent. However, the right panel uses the more traditional decibel scale where the resemblance to the traditional representations of the psychometric function is more evident.

\section{B. Threshold/duration functions}

We define threshold as the mean peak pressure when $\Psi=0.5$ and this is indicated by a horizontal line in the middle of the two panels in Fig. 1. The intersection of each function with this line represents the threshold for that particular duration. Clearly, longer durations are predicted to require lower pressures at threshold.

Thresholds can be found directly by substituting $\Psi=0.5$ in Eq. (3). After noting that $\ln (0.5)=-0.69$ and rearranging, we obtain the expression

$$
P_{\mathrm{thr}}=(0.69 / d+A) / G,
$$

where $P_{\text {thr }}$ is the peak pressure-at-threshold (in $\mu \mathrm{Pa}$ ). This is the function that predicts lower thresholds for longer durations. Figure 2 shows the predicted thresholds taken from Fig. 1 for each of the four durations. The continuous lines in Fig. 2 are the predicted thresholds computed using Eq. (4).

The result is presented twice; first using linear scales for pressure and time (left panel) and, second, on a log/log scale (dB SPL/ log duration) in the right panel. The dashed line in
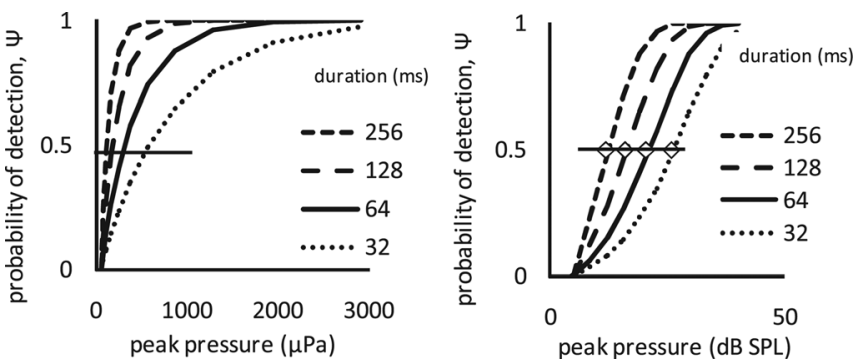

FIG. 1. Psychometric functions for four different tone durations. Equation (3) is used to generate the functions with $G=0.04$ events $/ \mathrm{s} / \mu \mathrm{Pa}$ and $A=2$ events/s. The duration, $d$, is specified in the legend. Left: the function is shown using micropascals for the abscissa. Right: same as left panel but the abscissa is given in decibels (dB SPL). Individual markers (unfilled diamonds) represent thresholds (50\% detection) at different tone durations. 

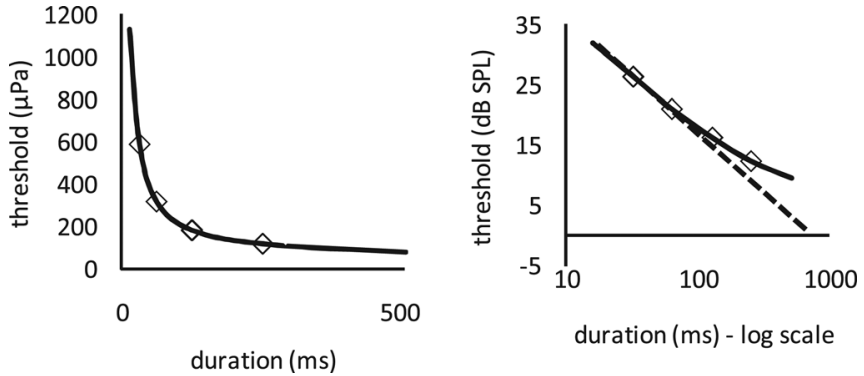

FIG. 2. Left panel: the unfilled diamonds are predicted thresholds for four tone durations taken from Fig. 1. The continuous line is based on Eq. (4) using parameters $G=0.04$ and $A=2$ and represents the predicted thresholds. Data points are plotted as peak pressure, $\mu \mathrm{Pa}$, versus tone duration (ms). Right panel: same as left panel except that thresholds are plotted as decibels (dB SPL) versus log (duration). The dashed line is a power function shown for comparison.

the right panel indicates a simple power function (see Introduction and, e.g., Zwislocki, 1960; Florentine et al., 1988). The probability-summation function and the power function are close except at long durations. Florentine et al. (1988) noted that shallower slopes at longer durations have been observed in some (but not all) psychophysical experiments and reports differ in terms of the duration at which the two functions diverge.

\section{Changing parameters}

The effects on the T/D function of changing parameters $G$ and $A$ are illustrated in Fig. 3 (left column). Reductions in the gain parameter, $G$, [while $A$ is held constant, Fig. 3(A)] produce parallel upward shifts in the T/D function. On the other hand, changes to the $A$ parameter [while $G$ is held constant, Fig. 3(B)] affect both the overall threshold and the size of the threshold difference between short and long tones.

Figure 3 (right column) shows the effect of parameter changes on the psychometric functions generated using Eq. (3). When $A$ is fixed, a reduction in the $G$ parameter results in a right shift of the psychometric function but the slope remains unchanged [Fig. 3(C)]. However, when the value of $A$ is increased (while holding $G$ constant), the psychometric function becomes steeper [Fig. 3(D)]. These observations lead to the important prediction that individuals who show only a small threshold difference in the T/D function should also have steeper psychometric functions.

The validity of the equations proposed above will be evaluated using human data from normal-hearing $(\mathrm{NH})$ and hearing-impaired listeners. Section III evaluates Eq. (4) using normal and impaired threshold/duration data. The experiment demonstrates that the equations outlined above are a good fit to the T/D data for both normal and impaired hearing. Section IV assesses Eq. (3) and the prediction of steeper psychometric functions in impaired listeners and also explores the relationship between the T/D measurements and psychometric slopes. By collecting both T/D and psychometric function data from the same subjects it becomes possible to correlate the two measures and test the theory.

When fitting the equations to the data, a least-squares, best-fit method will be used minimizing the error of prediction of thresholds expressed as decibels SPL. Alternatively,
T/D functions

\section{Psychometric functions}

\section{Variable $\boldsymbol{G}$}

A

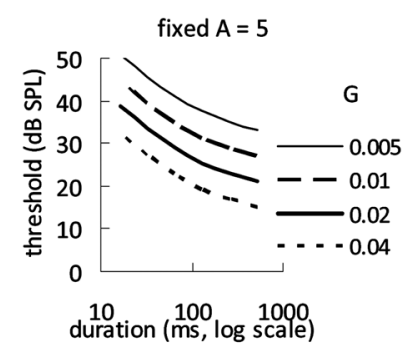

C

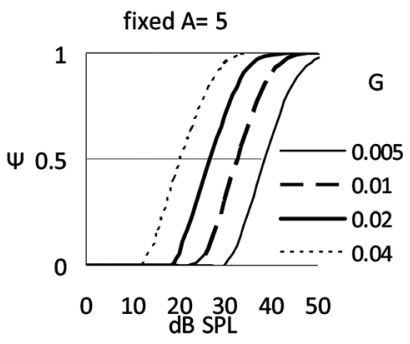

\section{Variable $A$}

$\mathbf{B}$

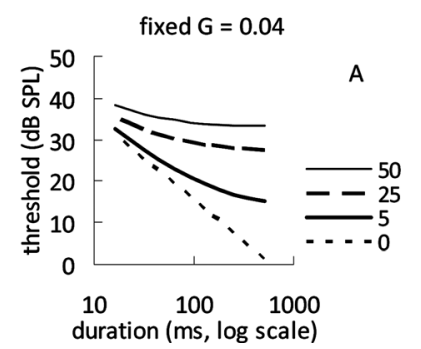

D

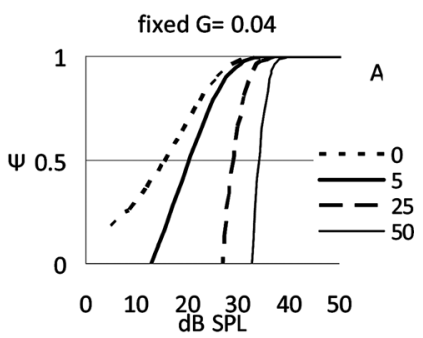

FIG. 3. Left column: predicted thresholds as a function of duration using Eq. (4). (A) The effect of changes to the $G$ (gain) parameter while fixing $A=5$. (B) The effect of changing the $A$ (sensory threshold) parameter while fixing $G=0.04$. Right column: psychometric functions for 0.1 s tones using Eq. (3). (C) Parameter $G$ is varied while $A$ is held constant at 5. (D) Parameter $A$ is varied between 0 and 50 while $G$ is held constant at 0.04 .

when only two thresholds are measured for a long and a short tone, the following Eqs. (5) and (6) can be used to estimate $A$ and $G$ directly. These formulas are derived from Eq. (4) by using two different and well-spaced durations $d_{1}$ and $d_{2}$ (in seconds) with their respective thresholds $\left(P_{1}\right.$ and $P_{2}$; the mean peak pressure in $\mu \mathrm{Pa}$ ). An example of the use of this procedure is given in the discussion but may be of use generally in clinical situations.

$$
\begin{aligned}
& A=0.69\left(P_{1} / d_{2}-P_{2} / d_{1}\right) /\left(P_{2}-P_{1}\right), \\
& G=\left(0.69 / d_{2}+A\right) / P_{2} .
\end{aligned}
$$

\section{EVALUATION: THRESHOLD/DURATION FUNCTIONS}

This section evaluates the equations with respect to the $\mathrm{T} / \mathrm{D}$ functions in two groups of listeners ( $\mathrm{NH}$ and hearingimpaired).

\section{A. Method}

\section{Procedure}

Absolute thresholds for a pure tone as a function of duration were measured in $\mathrm{NH}$ and hearing-impaired listeners. 
A single-interval, adaptive, up-down procedure was used throughout as described and evaluated by Lecluyse and Meddis (2009). Data were collected from eight NH and eight hearing-impaired listeners.

Data collection was divided into three identical blocks (replications). Each block consisted of 24 runs measuring pure-tone thresholds for four frequencies and six durations. The four test frequencies were presented in a random order within the block. Within a frequency sub-block, six-threshold measurements were made, one for each duration and these were also presented in random order. The data points to be presented are the mean of three measurements, one from each block.

At the beginning of each run, the level of the tone was set so that the participant could clearly hear it. This was a tone intensity of $40 \mathrm{~dB}$ SPL for normal listeners and, typically, $70 \mathrm{~dB}$ SPL for impaired listeners. If the participant indicated that a tone had been heard, the level was reduced on the next trial, otherwise it was increased. The initial step size was $10 \mathrm{~dB}$ but this was reduced to $2 \mathrm{~dB}$ after the first reversal. Each run consisted of 10 trials. The trial count starts from the presentation before the first reversal. Catch trials and initial trials are not included in the trial count.

Test tones were preceded by a cue tone consisting of a tone identical to the test tone but $10 \mathrm{~dB}$ more intense than the test tone. The participant's task was to count the number of tones heard and depress one of two buttons. If the " 2 " button was pressed, it was inferred that the test tone had been heard otherwise depression of the " 1 " (or "0") button was taken to mean that the test tone was not heard.

Twenty percent of trials were catch trials when only the cue tone was presented. If a "yes" response was given during a catch trial, the run was aborted, the participant informed, and the run was restarted. Participants were told that frequent catch trials would be presented and were encouraged not to make the mistake of reporting a stimulus when none was presented. Aborted runs were rare $(<5 \%)$.

A threshold estimate was based on all ten trials in the run (not including catch trials and initial trials). The threshold (dB SPL) was determined at the end of each run by fitting a logistic function to the data using the equation

$$
p(L)=\frac{1}{1+\mathrm{e}^{-k(L-\theta)}},
$$

where $p(L)$ is the proportion of yes-responses, $L$ is the level of the stimulus (dB SPL), $k$ is a slope parameter, and $\theta(\mathrm{dB}$ SPL) is the threshold to be estimated, i.e., the level of the stimulus at which the proportion of yes-responses is 0.5 . Parameters $k$ (slope) and $\theta$ (threshold) were obtained by minimizing the least-squares error.

\section{Stimulus}

Thresholds as a function of duration were measured for tones at 250,1000, 4000, and $8000 \mathrm{~Hz}$. Tone durations were $16,32,64,128,256$, and $512 \mathrm{~ms}$ (including 4-ms raised-cosine ramps). The cue tone preceded the test tone by $500 \mathrm{~ms}$. The cue/test tone pair was initiated under computer control
$0.5 \mathrm{~s}$ after the listener's previous response. When a catch trial occurred, only the cue tone was presented at the level appropriate for the following non-catch trial.

\section{Listeners}

Participants were eight NH listeners between the age of 20 and 35 yr and eight impaired-hearing ( $\mathrm{IH})$ listeners between the age of 40 and $67 \mathrm{yr}$. The IH listeners were volunteers with moderate to severe sensorineural hearing loss. All had a high frequency loss except for IH3 who had a cookie-bite (mid-frequency) loss around $1 \mathrm{kHz}$. This group was self-selected on the basis of their willingness to visit the laboratory regularly to help in developing a range of testing procedures. All listeners had been fitted with hearing aids at some time in the past. All were very experienced subjects by the time the data were collected for this study. None of the IH group showed any conduction losses as measured by bone conduction tests.

The NH listeners were also volunteers who either worked in the hearing laboratory or were student members of the Psychology department. None had a medical history of hearing problems. The majority were experienced listeners.

The left ear was tested in all cases where possible. The right ear was tested for four impaired listeners because their left ear had either normal or very high thresholds. All listeners received adequate training prior to the data collection. Thresholds for all participants are given in Fig. 4 for all four frequencies using 500 -ms tones.

\section{Apparatus}

Listeners were seated in a sound-proof booth, and stimuli were presented through circumaural headphones (Sennheiser HD600, Sennheiser electronic GmbH \& Co KG., Wedemark, Germany) linked directly to a computer sound card (Audiophile 2496, 24-bit, 96000-Hz sampling rate). Responses ( 1 or 2 or, occasionally, 0 ) were made using a button box. A monitor in front of the participant showed a display of the button box. While the stimulus was being presented, the symbols representing the buttons disappeared from the display. Immediately after stimulus presentation, the buttons reappeared on the screen to signal that a response was required. No feedback was given except immediately after being caught out on a catch trial.

\section{Analysis}

Two functions were fitted to the data separately for each individual at each frequency.

(1) Probability-summation. The T/D data were fitted with the probability-summation function given in Eq. (4) using a least-squares best-fit procedure minimizing the error of prediction of thresholds expressed as decibels SPL.

(2) Power function. A power function is a straight line on a $\mathrm{dB} / \log (d)$ plot and is a traditional way of representing this type of data (see, e.g., Garner, 1947; Zwislocki, 1960; Florentine et al., 1988; Gerken et al., 1990; and Neubauer and Heil, 2008).

$L_{\mathrm{thr}}=a \log (d)+b$, 

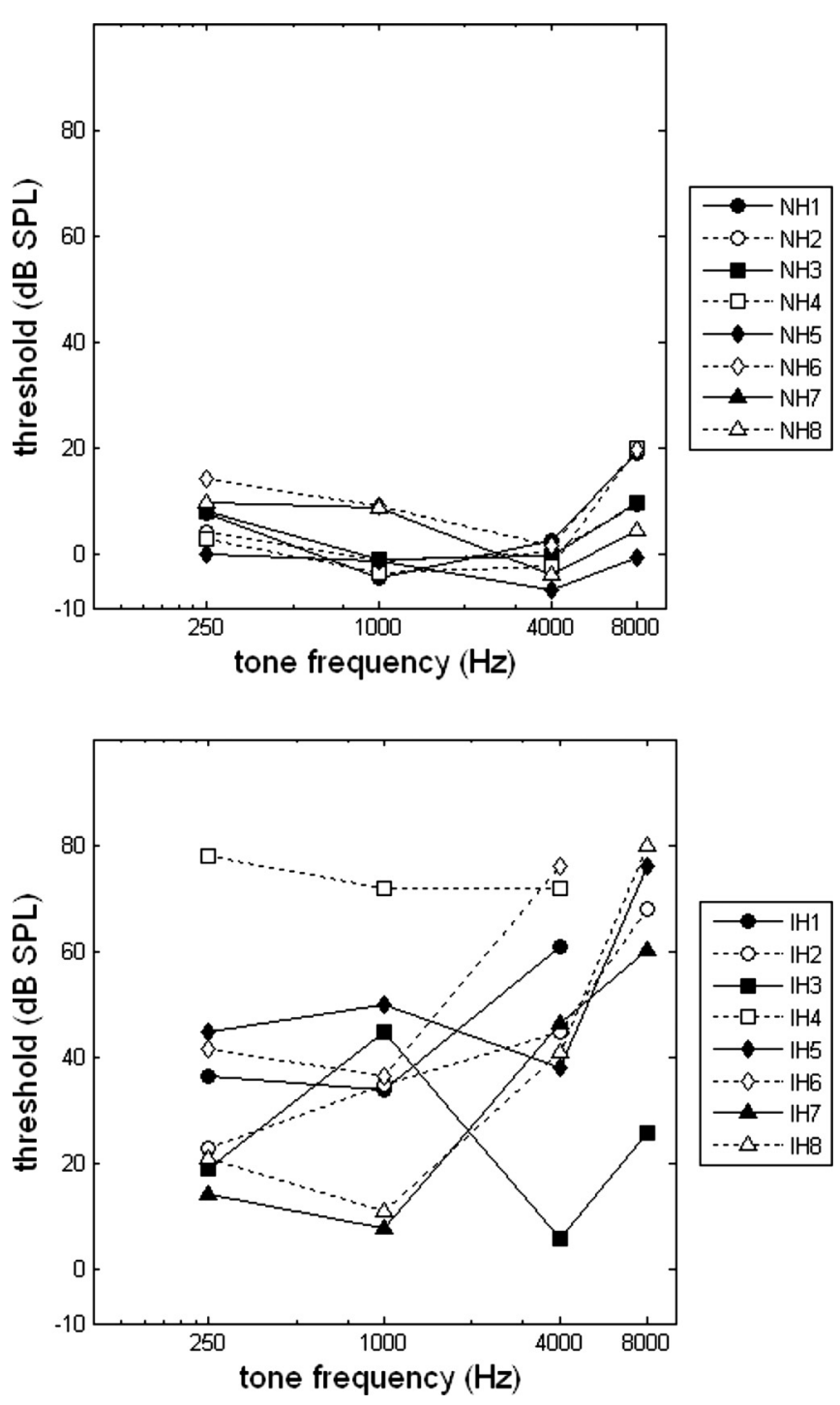

FIG. 4. Absolute thresholds for eight NH (top panel) and eight IH (bottom panel) participants.

where $L_{\mathrm{thr}}$ is the threshold expressed as decibels SPL, $d$ is the duration of the tone, and $a$ and $b$ are the free parameters. This function was fitted using a least-squares best-fit procedure minimizing the error of prediction of thresholds expressed as decibels SPL. The function was evaluated twice with and without a level correction for the 4-ms stimulus ramp.

\section{B. Results}

Figure 5 shows the T/D data for eight $\mathrm{NH}$ and eight $\mathrm{IH}$ listeners. No T/D function could be obtained for the 8000 $\mathrm{Hz}$ condition for three IH listeners (IH1, IH4, and IH6). As expected, almost all of the IH listeners show functions which are shallower than those of the NH listeners. The mean threshold difference between a 16-ms tone and a 512-ms tone is $9.8 \mathrm{~dB}$ (standard deviation, $\mathrm{SD}=2.8 \mathrm{~dB}$ ) in normal listeners and $4.3 \mathrm{~dB}(\mathrm{SD}=2.5 \mathrm{~dB})$ for the impaired listeners.

The continuous black lines in Fig. 5 show the best-fit functions using Eq. (4) for all listeners at the four test frequencies. The function provides a useful fit to the data in almost all cases yielding an average root mean square (rms) error of $0.86 \mathrm{~dB}$ for $\mathrm{NH}$ listeners and $0.71 \mathrm{~dB}$ for $\mathrm{IH}$ listeners.

The traditional power function fit represented by Eq. (8) also produced fits with average rms errors of 1.14 and 0.93 $\mathrm{dB}$ for the $\mathrm{NH}$ and $\mathrm{IH}$ groups, respectively. When the power function is fitted using thresholds that are corrected to take the tone ramps into account (i.e., using mean peak pressure), the fit is improved to rms errors of 0.91 and $0.71 \mathrm{~dB}$, respectively. This agrees with the finding of Gerken et al. (1990) that the power function fit is improved when the ramps are taken into account. We can conclude that the probabilistic formulation gives an equally good numerical account of the data as the traditional power function.

Average (median) estimated parameters for $A$ and $G$ for each frequency for both types of listeners are shown in Fig. 6 ( $\mathrm{NH}$ - continuous line, $\mathrm{IH}$-dashed line). Estimates for all $\mathrm{IH}$ listeners were only possible up to $4 \mathrm{kHz}$. Not surprisingly, the $G$ parameter, reflecting system gain, is reduced in the IH group. The IH group also shows higher estimates of $A$, particularly at high frequencies, reflecting an increase in the sensory threshold.

Estimates of parameters $A$ and $G$ are explored further in Fig. 7 using data from the NH group. The insets show the shared variances between the variables plotted. Except where specified all correlations were computed using logarithmic functions. In the top row, both $A$ and $G$ parameters are plotted against threshold difference (between 16 and 512 ms-thresholds). The $A$ parameter has, by far, the stronger association with threshold difference. In the second row, both $A$ and $G$ parameters are plotted against absolute threshold for a 128-ms tone. As expected there is a strong association between the $G$ parameter and threshold while $A$ is not correlated with threshold. In the bottom row of Fig. 7, $G$ is plotted against $A$ for the NH group only. The linear correlation between $A$ and $G$ estimates is low $\left(R^{2}=0.02\right)$ suggesting that the two parameters are largely independent.

The analysis in the figure is restricted to this NH group because a spurious correlation might be expected across the complete group of listeners if both parameters are affected by the impairment. The analysis was, however, repeated for the impaired group (not illustrated) where a similar pattern was found. Within this group, the linear correlation between the $A$ and $G$ estimates was also low $\left(R^{2}=0.02\right)$. Estimates of $A$ were more closely related to threshold difference $\left(R^{2}=0.61\right)$ than the absolute threshold $\left(R^{2}=0.20\right)$. By contrast, estimates of $G$ were more closely related to absolute threshold $\left(R^{2}=0.87\right)$ than threshold difference $\left(R^{2}=0.20\right)$.

From this, we may infer that the $A$ and $G$ parameters are largely independent that the $A$ parameter reflects the threshold difference between long and short durations tones and that the $G$ parameter is related to the average threshold. If this is the case, we might expect a shallow $\mathrm{T} / \mathrm{D}$ function to indicate a raised sensory floor.

The two-threshold estimates described in Eqs. (5) and (6) were evaluated across all listeners and all frequencies using only thresholds at 16 and $512 \mathrm{~ms}$. The formulas could not be used for eight T/D functions where the threshold difference for short and long durations was zero or negative. 

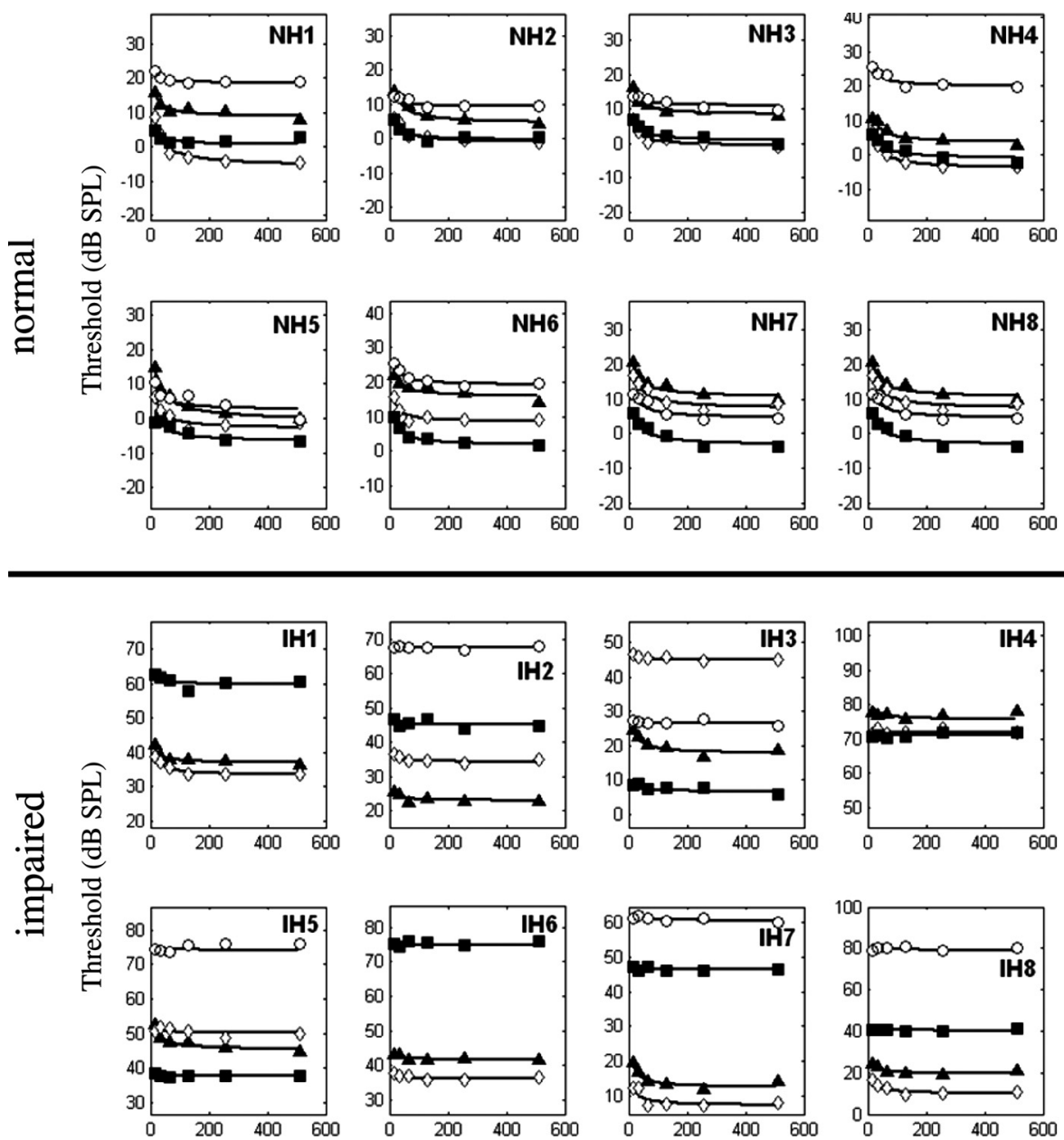

FIG. 5. Threshold/duration functions for NH listeners (top panel) and IH listeners (bottom panel). Data points represent thresholds (in $\mathrm{dB}$ SPL) at six durations (16-512 $\mathrm{ms}$ in octave steps, abscissa) at four different frequencies $(\mathrm{Hz}-250$, black triangles; 1000, white diamonds; 4000, black squares; and 8000, white circles). The continuous lines represent best-fit functions using Eq. (4). The ordinates always span $60 \mathrm{~dB}$ to facilitate visual comparisons of the size of the threshold difference. The exception is IH8 (bottom right) where the range of thresholds (across tone frequency) required a greater ordinate range.
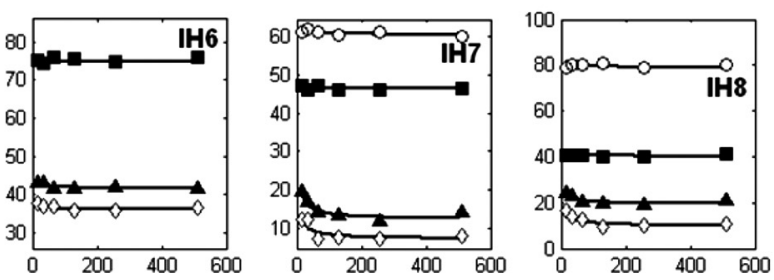

The remaining 53 estimates were found to be well correlated with the six-threshold estimates discussed above $\left(R^{2}=0.84\right.$ and 0.81 for the $G$ and $A$ estimates, respectively).

\section{EVALUATION: PSYCHOMETRIC FUNCTIONS}

The probability-summation equations offer a probabilistic explanation of the psychometric function for absolute threshold using Eq. (3). In particular, the probability-summation hypothesis predicts that the slope of the psychometric function will be steeper for some types of hearing impairment where the sensory threshold (parameter $A$ ) is raised. In
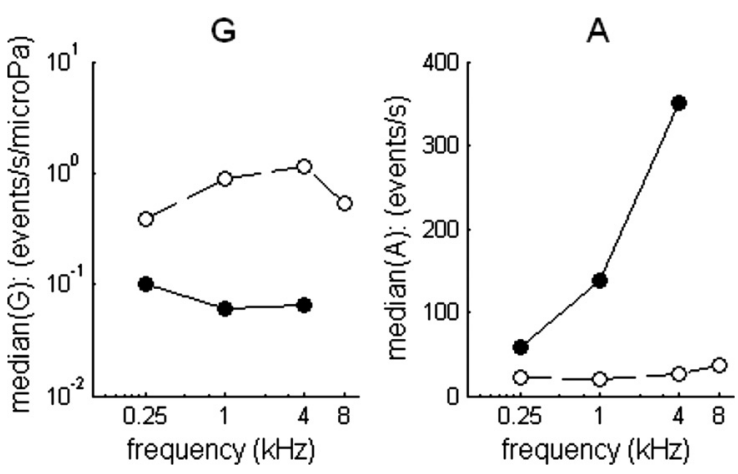

FIG. 6. Average estimates (median) for parameters $G$ and $A$ [see Eq. (4)] at different tone frequencies for $\mathrm{NH}$ (continuous line) and $\mathrm{IH}$ groups (dashed line). Estimates for IH listeners were only possible up to $4 \mathrm{kHz}$.
Sec. III, it was shown that listeners with sensorineural hearing loss had, as a group, higher average sensory thresholds and the prediction follows that they should also have steeper psychometric functions. Psychometric functions for $\mathrm{NH}$ and IH listeners were obtained in a second experiment to evaluate this hypothesis.

\section{A. Method}

\section{Participants}

Six of the eight IH participants who took part in experiment 1 took part again (IH1-IH6) and two more volunteers with sensorineural hearing loss (IH9 and IH10) were added to the participant pool. Two of the eight $\mathrm{NH}$ participants took part again (NH1, NH2) and six more participants with normal hearing (NH9-NH14) were added.

\section{Procedure and Stimuli}

The procedure for collecting psychometric functions was essentially the same as that described for experiment 1 except that all observations were made using $100-\mathrm{ms}, 1-\mathrm{kHz}$ pure tones. The same cued, single-interval, adaptive, one up/one down procedure was used throughout. One difference was that each run consisted of 30 trials rather than the ten trials used in the previous experiment. The other difference was that each participant was tested for 20 runs. This yielded a total of 600 responses in addition to catch trials. 

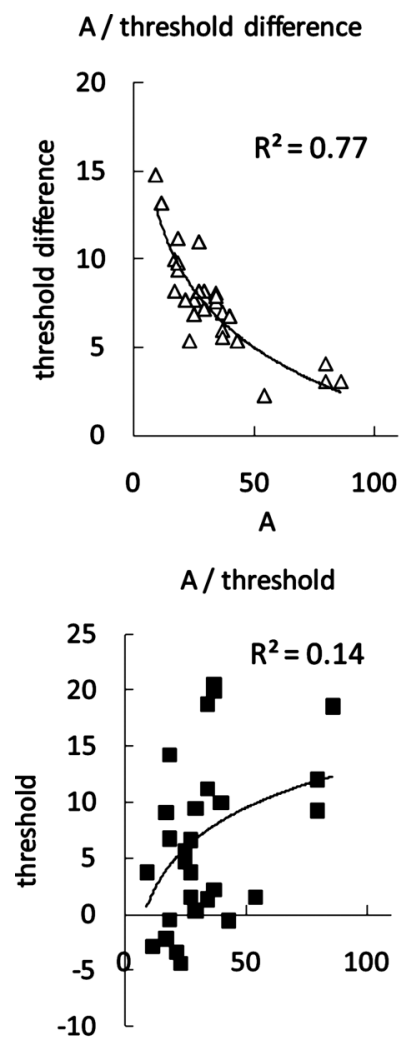

A

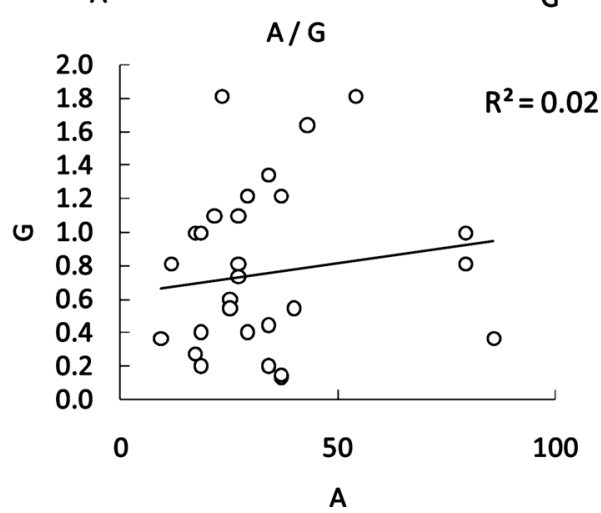

FIG. 7. Parameter estimates $A$ and $G$ for NH participants plotted against $\mathrm{T} / \mathrm{D}$ data characteristics irrespective of test frequency. Top row: estimates of $A$ (left) and $G$ (right) plotted against threshold difference. Middle row: estimates of $A$ (left) and $G$ (right) plotted against threshold. The threshold used is for the 128-ms duration. Bottom row: estimates of $A$ and $G$ plotted against each other. The correlations in the top two rows are computed using logarithmic functions; $y=a+b \log (x)$. In the bottom row, a linear relationship was assumed. Inset values are shared variances.

The data collection was divided into separate runs to allow rest intervals to be introduced. Typically this was a 2-min break after each third run. All runs were completed in the same session. All cue and test tones had 4-ms raised-cosine ramps as before.

Threshold/duration data were also collected from the new participants. The procedures used were the same as in experiment 1 .

\section{Statistical analysis}

For each participant, all 600 test observations were classified as either yes or "no" in a binary array, $\Psi$, and associated with the corresponding tone pressure level expressed either as $\mu \mathrm{Pa}, P$, or as decibels SPL, $L$.

A logistic function [Eq. (7)] was fitted to the data to give a conventional measure of the slope, $k$, of the function at threshold. The hypothesis to be tested was that the slope will be steeper (i.e., $k$ will be greater) for the IH group. In particular, it was expected that the slope would be most strongly associated with the increase in sensory threshold as revealed by the $T / D$ data. The equation was fitted using a least-squares best-fit procedure (minimising the error in predicting the probability of detection) using both corrected and uncorrected data. Data correction was achieved by subtracting the mean level for a run from each observation level in the run and subsequently adding back the overall threshold estimate for all of the listener's observations. Data correction was necessary to reduce the influence of variance associated with between-run threshold changes. Heil et al. (2006) showed that parallel shifts in the psychometric function do occur across repeated measurements and simple correction with respect to the mean value results in less variable functions. Threshold drift when uncorrected has the effect of reducing the slope and it was hoped to minimize this effect.

Best-fit psychometric functions for the probability-summation equation [Eq. (3)] to the corrected data are shown as dashed lines in Fig. 8 for each listener. The fit was obtained by minimizing the differences between the predicted and observed thresholds in decibels SPL. The rms error for the fits of the two functions (logistic and probability-summation) were almost exactly the same to two decimal places.

For the purposes of illustration only, the proportion of yes-responses was obtained for each level (1-dB bins) and presented in Fig. 8 as circles so that the height of a circle represents the proportion of yes-responses while the size of the circle represents the relative number of observations. It is in the nature of adaptive procedures that most observations are concentrated at levels close to threshold and the circles are larger in that region.

\section{B. Results}

The parameter $k$ of the best-fit logistic function (continuous lines in Fig. 8) was used to estimate the psychometric slope. When no correction was made for between-run threshold shifts, the fit of the logistic function was poor for a number of individuals. However, after correction, the fits were considerably better.

The IH group has steeper slopes on average $(M=1.14$; standard error, $\mathrm{SE}=0.08)$ than the $\mathrm{NH}$ group $(M=0.87$, $\mathrm{SE}=0.05)$ using corrected-level data $(t(14)=2.7, p<0.01$, one-tailed). The difference between the groups was less clear when uncorrected means are used; the mean of the IH group was $0.67(\mathrm{SE}=0.08)$ and that of the $\mathrm{NH}$ group was 0.47 $(\mathrm{SE}=0.06)(t(14)=1.9, p=0.04$, one-tailed $)$. The slope estimates are lower for the uncorrected data because the threshold variation between runs adds to the variability and hence increases the width of the psychometric function. The uncorrected data yield slope values that are consistent with those published previously (Watson et al., 1972; Green, 1993; Lecluyse and Meddis, 2009), i.e., in the region of 0.5. 

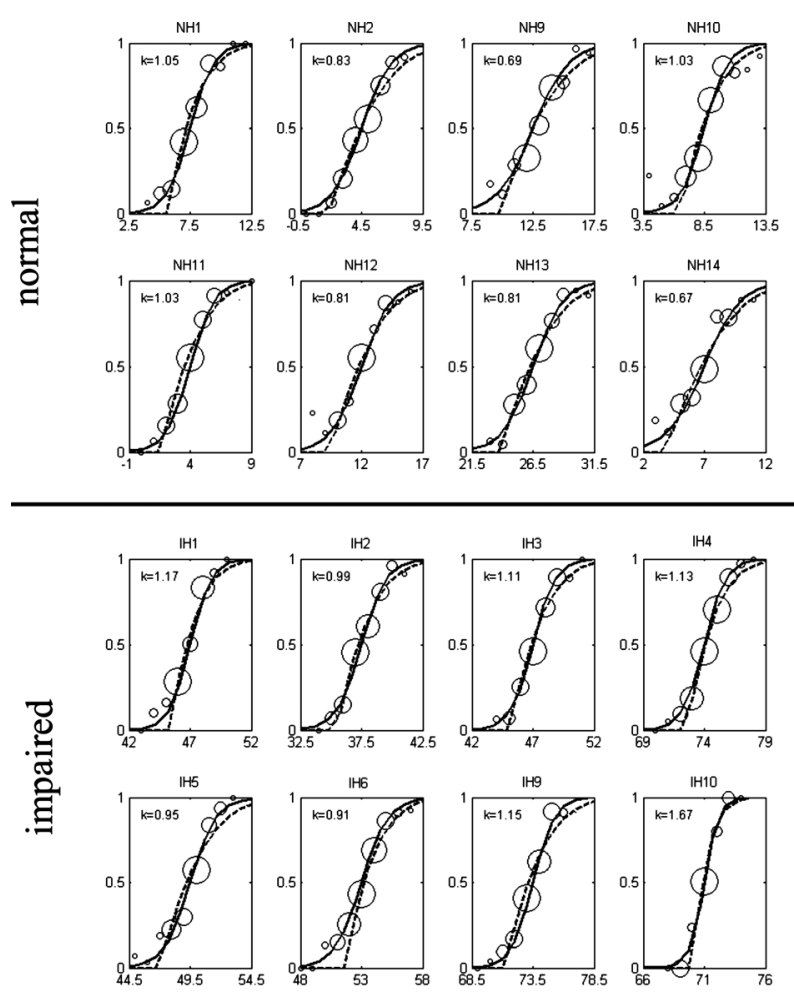

tone level (dB SPL)

FIG. 8. Psychometric functions for NH (top panel) and IH (bottom panel) participants using corrected data. The height of unfilled circles indicates the proportion of yes-responses ( $y$-axis) at a given tone level ( $x$-axis). The sizes of the circles indicate the relative number of observations obtained at that level. Least-squares best-fit probability-summation functions obtained using Eq. (3) are shown as dashed lines. Least-squares, best-fit logistic functions using Eq. (7) are shown as continuous lines. The slope, $k$, of the logistic function at threshold is specified as an inset in each figure. The abscissa (tone level, dB SPL) is arranged so that the estimated threshold for each data set defines the mid-point of the abscissa; e.g., IH9's threshold is 73.5 $\mathrm{dB}$ SPL. The width of each figure is always $10-\mathrm{dB}$ to facilitate visual comparison of slopes.

It should be noted that previous studies are likely to have been affected by threshold shifts during data collection even if this was not reported or corrected.

As expected, the slopes of psychometric functions of the $\mathrm{IH}$ group were, on average, steeper than those of the $\mathrm{NH}$ group. However, some overlap was present between the groups, and caution is indicated in the interpretation of the results. A similar result was reported by Arehart et al. (1990). The data do not justify a blanket conclusion that impairment is always associated with abnormally steep psychometric functions. Instead, we should consider the more detailed prediction of the probability-summation hypothesis that steeper functions are associated with listeners who show specific symptoms of an increase in the sensory threshold.

A correlation analysis was performed to investigate the relationship between the psychometric slope, $k$, and probability-summation parameters $A$ and $G$ obtained using the $\mathrm{T} / \mathrm{D}$ data for $1-\mathrm{kHz}$ tones only. The relationship between the estimated psychometric slopes and the parameters $A$ and $G$ derived from the T/D data is complicated by the fact that the $A$ and $G$ parameters are correlated with each other when normal and impaired data are pooled. This is because impair- ment is associated with changes in both parameters for some impaired listeners. The Pearson product moment correlation coefficient, $r$, was found to be -0.44 between $A$ and $G$ when computed across all subjects. This value was used to compute partial correlation coefficients. The partial correlation between $A$ and the slope, $k$, (after partialling out $G$ ) was 0.65 while the partial correlation between $G$ and $k$ (after partialling out $A$ ) was only -0.27 . This result is consistent with the prediction based on the probability-summation equations [see Figs. 3(C) and (D)]. It implies that the steepness of the psychometric function is related to the height of the sensory threshold rather than the gain of the system.

\section{DISCUSSION}

The project has four principal findings: (1) the probability accumulation equations [Eqs. (3) and (4)] proved to be a good fit to both the T/D and psychometric function data for NH and hearing-impaired listeners; (2) the prediction of a numerical link between the T/D and psychometric functions was also supported by the data; (3) the key parameters, gain $(G)$ and sensory threshold $(A)$ are likely to be independent; and (4) simplified equations [Eqs. (5) and (6)] permitting rapid estimation in a clinical context based on only two-threshold estimates were useful estimates of parameters $A$ and $G$.

Before discussing the findings in detail, it is necessary to outline the similarities and differences with the considerable body of theoretical and empirical work already published in this area by Heil and Neubauer (2001, 2003), Heil et al. (2008), and Neubauer and Heil (2004, 2008). The similarities are most obvious in our basic assumptions that (1) psychophysical thresholds can be understood in terms of low-probability detection events and (2) these can be mathematically described by the accumulation of probabilities rather than the accumulation and dissipation of energy or information in some kind of integrator or memory.

Heil and Neubauer (2003) argue that these principles can be observed in action as early as the IHC/AN synapse and, as a consequence, this must be the source of the psychophysical T/D function. It could equally well be argued, however, that the same process is operating throughout the nervous system whenever the timing of a critical event is uncertain. If this is the case, later events in the signal processing sequence could prove to be more relevant. This was illustrated in a computer model of the auditory periphery that has been shown to reproduce both their first-spike latency data and appropriate psychophysical thresholds (Meddis, 2006) based on coincidence detection at a later stage. For this reason, we submit that it is premature to identify the probability accumulation process with a single physiological location. Nevertheless, it is useful to preserve the general probability accumulation principle for psychophysics as a process with the power to explain "temporal integration" without reference to a physical integrator. In the presentation of the equations, care has been taken to avoid any identification with specific physiological processes. This allows important issues to be pursued in the psychophysics independently 
of the physiology. Two such purely psychophysical topics are discussed above: (1) the relationship between T/D and psychometric functions and (2) changes in the shape of the $\mathrm{T} / \mathrm{D}$ function that accompany hearing impairment.

A further difference in our approach can be found also at the detailed level of the derivation of the equations. Heil and Neubauer have concentrated on the time delay (related to the stimulus pressure integral) between the stimulus onset and the first physiological event signifying detection. Our equations are a solution to the simpler mathematical problem of specifying the likelihood of observing at least one detection event (that could occur at any time) before the end of the stimulus. The resulting equation for the $\mathrm{T} / \mathrm{D}$ function requires only two parameters and is quite distinct from the traditional power function. The physiological approach, in contrast, produces a system of equations with a larger number of intrinsic parameters. Neubauer and Heil (2004) use the power function $\left(k . d^{-m}\right.$, Green et al., 1957) as their main tool for describing psychophysical thresholds. At first sight, this is a model that requires only two parameters gain, $k$, and slope, $m$. They even argue that the slope parameter, $m$, is fixed around a value of 0.667 by the physiology of the IHC calcium dynamics and thus the number of free parameters is reduced. However, their power function contains additional parameters because stimulus duration, $d$, in the formulation of Green et al. (1957) is replaced by a new concept, the "effective duration;" i.e., the total time when the stimulus pressure is above some minimum value. This more sophisticated definition of duration produces the required improvement of fit to the data at longer tone durations. However, the effective duration depends on an additional parameter, relating to "effective pressure."

Another difference can be seen in the choice of a parameter to determine the sensory threshold. Neubauer and Heil (2004) specify a "minimum effective pressure," $P_{\text {ineff }}$, while we propose a "minimum event rate," $A$. Our choice of this parameter is deliberate and intended to allow parameters $G$, and $A$ to be independent. If we use the minimum effective pressure concept, Eq. (1) becomes $G\left(P-P_{\text {ineff }}\right)$ or $G P-G P_{\text {ineff }}$ and both terms are modified by gain. Our preference for parameter $A$, the discounted event rate, was based on the desirability of decomposing threshold into potentially independent dimensions. The lack of a correlation between $A$ and $G$ in our data analysis suggests that this approach was successful.

While it is clearly necessary to explain the key differences between our formulations and those of Heil and Neubauer, it would miss the point of our general argument to emphasize them unduly. Our main concern is to exploit the opportunity afforded by their work to change the consensus of the last $50 \mathrm{yr}$ that thresholds must be understood in terms of a physical temporal integrator. The concept of absolute threshold is basic to hearing science and introducing the alternative notion of probability accumulation to auditory science is potentially a major development, and this is the central message of our report.

Some caveats need to be entered concerning the basic rate equation [Eq. (1)]. This assumes that the probability of an event increases linearly with pressure and that the pres- sure is constant throughout the stimulus. For more general applications, Watson's (1979) basic equation, $P=1$ $-\Pi\left(1-p_{t}\right)$, should be used. It allows the probability of a detection event to be specified for each instant in time dependent on some rule relating stimulus level to the probability of an event. This more general formulation may be necessary to take into account special factors such as the possibility of a non-linear response to stimulus level, a variable pressure envelope, the increased spread of excitation along the basilar membrane as the pressure-at-threshold rises between the longest and shortest tone durations and issues associated with threshold microstructure (e.g., Cohen, 1982). For example, Neubauer and Heil (2008) and Heil et al. (2008) propose a non-linear (third power) relationship between probability of events and the low-pass filtered stimulus amplitude but otherwise the ideas are very similar.

The assumption of a linear response near threshold has already been considered by Plack et al. (2004) and Plack and Skeels (2007) who estimated the cochlear input/output response function in listeners with both normal and impaired hearing. In both cases, they found that a linear response was to be expected in the region immediately above threshold. Plack and Skeels (2007) have also shown that the differences in the T/D function between normal and impaired listeners cannot be attributed to differences in stimulus compression. However, linearity may only apply in a narrow region of levels above threshold. At higher levels, compression must be taken into account. This is a particular problem for very short signals where the tone level required to exceed threshold may lie in the compressed region. To avoid this problem in our experiments, the tone durations in experiment I were never shorter than $16 \mathrm{~ms}$ and the range of thresholds was never greater than $20 \mathrm{~dB}$. Green et al. (1957) observed that the power function needed adjustment for very short stimuli. This problem has traditionally been explained in terms of spectral splatter that increases as signals are shortened. However, recent research on the threshold for stimulus compression (e.g., Plack et al., 2004) suggests that the high signal levels required to detect very short stimuli may lie inside the compression region leading to higher thresholds than predicted by an assumption of a linear response.

Plack and Skeels (2007) have also discussed the complications caused by threshold micro-structure, i.e., differences in sensitivity to tones that are close in frequency caused by possible resonances in the cochlea acting as a sort of comb filter. This will interact with tone duration in that the effect of spectral splatter associated with very short tone durations will differ according to whether the tone frequency lies near a valley or a peak of this filtering function. When the tone duration is reduced, the energy associated with the spectral splatter will increase and spread into these adjacent regions resulting in a rise or a fall in threshold, respectively. When splatter is likely to be an important component, it will be necessary to model this function explicitly before using Watson's equation. Hearing-impaired listeners are known to show much reduced microstructure (Mauermann et al., 2004) and this might have some bearing on the size of the effect when comparing threshold/duration functions of $\mathrm{NH}$ and IH listeners. 
It could be argued that the tripartite theory (noise, criterion, and integrator) is to be preferred because it also deals with thresholds for tones in noise. An effective rebuttal of this argument will need to be based on a probabilistic account that can also give an account of simultaneous masking. We are not yet able to give such an account. However, it is possible that Viemeister and Wakefield's (1991) theory can be amended to meet this requirement. Currently, it integrates information over the duration of the stimulus and uses the summed information to make a final decision about the presence or absence of a tone. If the theory were remodeled so that each separate "look" produced an immediate binary (yes/no) decision, the theory would reduce to one where the final decision was a yes if at least one of the individual looks produced a positive response. This would remove the need for an integrator and the theory would be reduced to a probabilistic formulation able to account for many phenomena observed in simultaneous masking without invoking an integrator.

Dai and Wright (1995) challenged the signal detection/temporal integration approach with a demonstration that the number of correct detections for masked tones near threshold depended on the listener's expectation of the duration of the tone. If their results could be replicated for tones in quiet, they would also challenge our approach. Their work highlights a general need to link up sensory theories with psychological theories concerning how attention is directed and sustained to take account of some and ignore other information. For example a "continuous look" model should respond to a short tone stimulus no matter when it occurs and whatever its expected duration. The matter calls for further investigation.

These detailed considerations will typically not apply in a clinical situation where steady fixed-duration tones are used and the differences between individuals are substantial. It is in this context that the idea of "threshold decomposition" may have most potential. Pure-tone audiometry forms the bed-rock of clinical assessment even though there is a general awareness that similar audiograms may conceal basic differences in the underlying pathology in patients with sensorineural hearing disorders. Decomposition of a patient's thresholds into two orthogonal components offers a potential basis for distinguishing groups of patients either as an indicator of pathology or as a predictor of treatment outcomes. It should certainly act as a caution against studying groups of undifferentiated cases of "impaired hearing." Equations (5) and (6) offer a quick method of assessment that need not extend the patient testing time unduly.

The probability accumulation theory predicts that the steepness of the psychometric function must be related to the $A$ parameter rather than gain; the higher the value of $A$, the steeper the psychometric function. This was indeed found to be the case across the $\mathrm{NH}$ and $\mathrm{IH}$ participants in the study. Carlyon et al. (1990) and Arehart et al. (1990) have already demonstrated some steeper psychometric function slopes associated with hearing impairment, and it is well known that impairment is often associated with changes in the T/D function. The novelty in our results is the confirmation that the reduction in the $\mathrm{T} / \mathrm{D}$ effect is better predicted by changes in the sensory floor parameter than changes in the gain parameter or any overall threshold measurement. Plack and Skeels (2007) have already indicated that the model of Neubauer and Heil (2004) can be interpreted as implying steeper functions in hearing-impaired listeners when the sensory threshold is raised. Our results support this contention on the basis of differences between individuals rather than simply between normal and impaired listeners.

Jerger (1955) found that the temporary threshold shift induced by 2 -min exposure to $110 \mathrm{~dB}$ SPL wideband noise raised thresholds more for long tones than short tones. This pattern is a symptom of raised sensory threshold rather than loss of gain [see Fig. 3(B)]. The mean threshold for a 5 and $500-\mathrm{ms}, 4-\mathrm{kHz}$ pure tones was reported to be 28 and $15 \mathrm{~dB}$ SPL, respectively, before exposure. Thirty seconds after exposure, the thresholds were 34 and $27 \mathrm{~dB}$ SPL. If we estimate $A$ and $G$ using Eqs. (5) and (6), we find that $G$ changes only marginally from 0.08 to 0.05 while $A$ increases threefold from 10.7 to 32.4 event/s. This suggests that a noiseinduced temporary threshold shift can be decomposed to expose a shift in the sensory floor but little change in the gain. During recovery, the $A$ parameter gradually returns to its preexposure value. In contrast, Neubauer and Heil (2004) compared thresholds in cats before and after noise trauma consisting of exposure for $48 \mathrm{~h}$ to a $2-\mathrm{kHz}$ tone at $110 \mathrm{~dB}$ SPL. The average thresholds before exposure were -4 and $-19 \mathrm{~dB}$ SPL for a 8-ms and a 275-ms tone, respectively, and 22 and $14 \mathrm{~dB}$ SPL after exposure. When our equations are applied to their data, we find a substantial reduction in $G$ (from 2.9 to 0.2 ) and a large rise in $A$ (from 7.7 to 26.3). This agrees with their conclusion that both gain and sensory floor are affected by sustained exposure to very loud sound. The difference between the two studies suggests that gain is not affected during temporary threshold shift but that permanent threshold shift associated with severe noise trauma is more complex. While this is an empirical issue, it highlights the conceptual issue that it is more profitable to think of threshold as a twovalued function and it is necessary, when describing a shift, to specify which of the two components of threshold are affected by a particular procedure.

Neubauer and Heil (2004) identify a rise in the sensory threshold with a rise in the ineffective pressure $\left(P_{\text {ineff }}\right)$ while our approach would characterize the change as a rise in the discounted rate, $A$. Pressure is an obvious metric, while discounted rate may seem to be more mysterious. However, it can be illustrated as an abstract principle in terms of coincidence detection. Here, a device discounts input pulses from a number of sources unless they arrive at approximately the same time. To achieve its goal of identifying only coincidental inputs, some rejection criterion needs to be set. This rejection level would correspond to the $A$ parameter or sensory threshold concept. The auditory nervous system may be characterized by many layers of coincidence detection, and the $A$ parameter in our model probably refers to an aggregate feature of the system's global operation. Whether the discounted rate is influenced by gating mechanisms in the IHC/AN synapse, feedback inhibition or long-term plasticity is a matter for physiology while the threshold characteristics of the resulting overall system is a matter for psychophysics. 
However, it now appears to be the case that it is not essential to resort to physical integrators or unceasing internal noise to explain the data in either physiology or psychophysics.

\section{ACKNOWLEDGMENTS}

This work was supported by a grant from the Engineering and Physical Sciences Research Council. The authors thank Chris Plack and two anonymous reviewers for their insightful comments on earlier versions of this manuscript.

Arehart, K., Burns, E. M., and Schlauch, R. S. (1990). “A comparison of psychometric functions for detection in normal hearing and hearing impaired listeners," J. Speech Hear. Res. 33, 433-439.

Carlyon, R. P., Buus, S., and Florentine, M. (1990). “Temporal integration of trains of tone pulses by normal and by cochlearly impaired listeners," J. Acoust. Soc. Am. 87, 260-268.

Chung, D. Y. (1982). "Temporal integration-its relationship with noiseinduced hearing loss," Scand. Audiol. 11, 153-157.

Chung, D. Y., and Smith, F. (1980). "Quiet and masked brief-tone audiometry in subjects with normal hearing and with noise-induced hearing loss," Scand. Audiol. 9, 43-47.

Clock, A. E., Salvi, R. J., Saunders, S. S., and Powers, N. L. (1993). "Neural correlates of temporal integration in the cochlear nucleus of the chinchilla," Hear. Res. 71, 37-50.

Clock, A. E., Salvi, R. J., Wang, J., and Powers, N. L. (1998). "Threshold duration functions of chinchilla auditory nerve fibers," Hear. Res. 119, 135-141.

Cohen, M. F. (1982). "Detection threshold microstructure and its effect on temporal integration data," J. Acoust. Soc. Am. 71, 405-409.

Dai, H., and Wright, B. A. (1995). "Detecting signals of unexpected or uncertain durations," J. Acoust. Soc. Am. 98, 798-806.

Elliott, L. L. (1963). "Tonal thresholds for short-duration stimuli as related to subject hearing level," J. Acoust. Soc. Am. 35, 578-580.

Florentine, M., Fastl, H., and Buus, S. (1988). "Temporal integration in normal hearing, cochlear impairment and impairment simulated by masking," J. Acoust. Soc. Am. 84, 195-203.

Garner, W. R. (1947). "The effect of frequency spectrum on temporal integration of energy in the ear," J. Acoust. Soc. Am. 19, 808-815.

Garner, W. R., and Miller, G. A. (1947). "The masked threshold of pure tones as a function of duration," J. Exp. Psych. 37, 293-303.

Gengel, R. W., and Watson, C. S. (1971). "Temporal integration: I. Clinical implications of a laboratory study. II. Additional data from hearingimpaired subjects," J. Speech Hear. Disord. 36, 213-244.

Gerken, G. M., Bhat, V. K. H., and Hutchison-Clutter, M. (1990). "Auditory temporal integration and the power function model," J. Acoust. Soc. Am. 88, 767-778.

Green, D. M. (1993). "A maximum-likelihood method for estimating thresholds in a yes-no task," J. Acoust. Soc. Am. 93, 2096-2105.

Green, D. M., Birdsall, T. G., and Tanner, W. P. (1957). "Signal detection as a function of signal intensity and duration," J.Acoust. Soc. Am. 29, 523-531.

Hall, J. W., and Fernandes, M. A. (1983). "Temporal integration, frequency resolution, and off-frequency listening in normal-hearing and cochlearimpaired listeners," J. Acoust. Soc. Am. 74, 1172-1177.

Harris, J. D., Haines, H. L., and Myers, C. K. (1958). "Brief tone audiometry," Arch. Otolaryngol. 67, 699-713.

Heil, P., and Neubauer, H. (2001). "Temporal integration of sound pressure determines thresholds of auditory-nerve fibers," J. Neurosci. 21, 7404 7415.

Heil, P., and Neubauer, H. (2003). "A unifying basis of auditory thresholds based on temporal summation," Proc. Natl. Acad. Sci. U.S.A. 100, 61516156.

Heil, P., Neubauer, H., Brown, M., and Irvine, D. R. F. (2008). "Towards a unifying basis of auditory thresholds: Distributions of the first-spike latencies of auditory-nerve fibers," Hear. Res. 238, 25-38.

Heil, P., Neubauer, H., Tiefenau, A., and von Specht, H. (2006). "Comparison of absolute thresholds derived from an adaptive forcedchoice procedure and from reaction probabilities and reaction times in a simple reaction time paradigm," J. Assoc. Res. Otolaryngol. 7, 279-298.
Hoglund, E. M., and Feth, L. L. (2009). "Spectral and temporal integration of brief tones," J. Acoust. Soc. Am. 125, 261-269.

Hughes, J. W. (1946). "The threshold of audition for short periods of stimulation," Proc. R. Soc. London Ser. B 133, 486-490.

Jerger, J. F. (1955). "Influence of stimulus duration on the pure-tone threshold during recovery from auditory fatigue," J. Acoust. Soc. Am. 27, 121124.

Krishna, B. S. (2002). “A unified mechanism for spontaneous-rate and firstspike timing in the auditory nerve," J. Comp. Neurosci. 13, 71-91.

Lecluyse, W., and Meddis, R. (2009). "A simple single-interval adaptive procedure for estimating thresholds in normal and impaired listeners," J. Acoust. Soc. Am. 126, 2570-2579.

Licklider, J. C. R. (1951) "Basic correlates of the auditory stimulus," in Handbook of Experimental Psychology, edited by S. S. Stevens (Wiley, New York), pp. 985-1039.

Mauermann, M., Long, G. R., and Kollmeier, B. (2004). "Fine structure of hearing threshold and loudness perception," J. Acoust. Soc. Am. 116, 1066-1080.

Meddis, R. (2006). "Auditory-nerve first-spike latency and auditory absolute threshold: A computer model," J. Acoust. Soc. Am. 119, 406-417.

Neubauer, H., and Heil, P. (2004). "Towards a unifying basis of auditory thresholds: The effects of hearing loss on temporal integration reconsidered," J. Assoc. Res. Otolaryngol. 5, 436-458.

Neubauer, H., and Heil, P. (2008). "A physiological model for the stimulus dependence of first-spike latency of auditory-nerve fibers," Brain Res. 1220, 208-223.

Olsen, W. O., and Carhart, R. (1966). "Integration of acoustic power at threshold by normal hearers," J. Acoust. Soc. Am. 40, 591-599.

Olsen, W. O., Rose, D. E., and Noffsinger, D. (1974). "Brief tone audiometry with normal, cochlear, and eighth nerve tumor patients," Arch. Otolaryngol. 99, 185-189.

Pedersen, C. B., and Elberling C. (1973). "Temporal integration of acoustic energy in patients with presbyacusis," Acta Otolaryngol. 75, 32-37.

Pedersen, C. B., and Salomon, G. (1977). "Conductive hearing loss evaluated by brief-tone audiometry," Acta Otolaryngol. 83, 424-428.

Plack, C. J., Drga, V., and Lopez-Poveda, E. A. (2004). "Inferred basilarmembrane response functions for listeners with mild to moderate sensorineural hearing loss,” J. Acoust. Soc. Am. 115, 1684-1695.

Plack, C. J., and Skeels, V. (2007). "Temporal integration and compression near absolute threshold in normal and impaired ears," J. Acoust. Soc. Am. 122, 2236-2244.

Plomp, R., and Bouman, M. A. (1959). "Relation between hearing threshold and duration for tone pulses," J. Acoust. Soc. Am. 31, 749-758.

Richards, A. M., and Dunn, J. (1974). "Threshold measurement procedures in brief-tone audiometry," J. Speech Hear. Res. 11, 446-454.

Sanders, J. W., and Honig, E. A. (1967). "Brief tone audiometry, results in normal and impaired ears," Arch. Otolaryngol. 85(6), 640-647.

Sheeley, E. C., and Bilger, R. C. (1964). "Temporal integration as a function of frequency," J. Acoust. Soc. Am. 36, 1850-1857.

Solecki, J. M., and Gerken, G. M. (1990). "Auditory temporal integration in the normal-hearing, hearing-impaired cat,” J. Acoust. Soc. Am. 88, 779785 .

Viemeister, N. F., and Wakefield, G. H. (1991). "Temporal integration and multiple looks," J. Acoust. Soc. Am. 90, 858-856.

Watson, A. B. (1979). "Probability accumulation over time," Vision Res. 19, 515-522.

Watson, C. S., and Gengel, R. W. (1969). "Signal duration and signal frequency in relation to auditory sensitivity," J. Acoust. Soc. Am. 46, 989997.

Watson, C. S., Franks, J. R., and Hood, D. C. (1972). "Detection of tones in the absence of external masking noise. I. Effects of signal intensity and signal frequency," J. Acoust. Soc. Am. 52, 633-643.

Wright, H. N. (1968). "Clinical measurement of temporal auditory summation,” J. Speech Hear. Res. 11, 109-127.

Wright, H. N. (1978). "Brief tone audiometry," in Handbook of Clinical Audiology, 2nd ed., edited by J. Katz (Williams and Wilkins, Baltimore), pp. 218-232.

Young, L. M., and Kanofsky, P. (1973). "Significance of brief-tone audiometry," J. Aud. Res. 13, 14-25.

Zwislocki, J. (1960). "Theory of temporal auditory summation," J. Acoust. Soc. Am. 32, 1046-1060. 